\title{
SCIENTIFIC REPORTS

\section{Efficacy of three-dimensionally printed polycaprolactone/beta tricalcium phosphate scaffold on mandibular reconstruction}

\begin{abstract}
Sanghoon Lee ${ }^{1,2}$, Dami Choi ${ }^{3}$, Jin-Hyung Shim ${ }^{3,4}$ \& Woong Nam (1) ${ }^{1 *}$
It has been demonstrated that development of three-dimensional printing technology has supported the researchers and surgeons to apply the bone tissue engineering to the oromandibular reconstruction. In this study, poly caprolactone/beta tricalcium phosphate (PCL/ $\beta-\mathrm{TCP})$ scaffolds were fabricated by multi-head deposition system. The feasibility of the three-dimensionally (3D) -printed PCL/ $\beta$-TCP scaffolds for mandibular reconstruction was examined on critical-sized defect of canine mandible. The scaffold contained the heterogeneous pore sizes for more effective bone ingrowth and additional wing structures for more stable fixation. They were implanted into the mandibular critical-sized defect of which periosteum was bicortically resected. With eight 1-year-old male beagle dogs, experimental groups were divided into 4 groups ( $n=4$ defects per group, respectively). (a) no further treatment (control), (b) PCL/ $\beta$-TCP scaffold alone (PCL/TCP), (c) PCL/3-TCP scaffold with recombinant human bone morphogenetic protein-2 (rhBMP-2) (PCL/TCP/BMP2) and (d) PCL/ $\beta$-TCP scaffold with autogenous bone particles (PCL/TCP/ABP). In micro-computed tomography, PCL/TCP/BMP2 and PCL/TCP/ ABP groups showed significant higher bone volume in comparison to Control and $\mathrm{PCL} / \mathrm{TCP}$ groups $(\mathrm{P}<0.05)$. In histomorphometric analysis, a trend towards more bone formation was observed in PCL/TCP/BMP2 and $P C L / T C P / A B P$ groups, but the results lacked statistical significance $(P=0.052)$. Within the limitations of the present study, 3D-printed PCL/3-TCP scaffolds showed acceptable potential for oromandibular reconstruction.
\end{abstract}

Since the introduction of microvascular free tissue transfer, vascularized bone graft (VBG) has become the treatment of choice for oromandibular reconstruction ${ }^{1,2}$. However, microvascular free tissue transfer might present surgical results with large variation depending on the surgeon's skill and experience. It also requires considerable hospital resources including two surgical teams, an intensive care unit and prolonged hospital stay. Furthermore, donor-site morbidity may lead to important aesthetic, functional, and psychosocial issues for patients. To overcome the heavy burden of free tissue transfer, maxillofacial surgeons have turned to the field of tissue engineering for reconstruction ${ }^{3-5}$, of which potential advantages may include more customized reconstruction, shortened operation time, rapid recovery and minimization of donor-site morbidity ${ }^{2}$.

In recent years, the development of three-dimensional printing (3DP) technology for tissue engineering has contributed to increased speed and precision from planning to operation in maxillofacial reconstruction 6 . 3DP technology has been researched in manufacturing scaffolds with growth factor in conjunction with a surgical guide and template. It is capable of not only reproducing the individual anatomical complexity of a maxillofacial defect but also optimizing functions and mechanical properties through the combination of various materials, stem cells and growth factors ${ }^{4,7,8}$. Several in vivo and in vitro studies involving 3DP, particularly using a polycaprolactone $/ \beta$-tricalcium phosphate (PCL/ $\beta$-TCP) combination, have shown the feasibility of scaffolds ${ }^{9,10}$. Finally, although clinical application of 3DP in maxillary reconstruction has been reported in the Republic of Korea ${ }^{11,12}$,

\footnotetext{
${ }^{1}$ Department of Oral and Maxillofacial Surgery, Yonsei University College of Dentistry, 50-1, Yonsei-ro, Seodaemoon-gu, Seoul, 03722, Republic of Korea. ${ }^{2}$ Department of Oral and Maxillofacial Surgery, Jukjeon Dental Hospital, College of Dentistry, Dankook University, 152, Jukjeon-ro, Suji-gu, Yongin-si, Gyeonggi-do, 16890, Republic of Korea. ${ }^{3}$ Research Institute, T\&R Biofab Co. Ltd., 237 Sangidaehak-Ro, Siheung-si, Gyeonggi-do, 15073, Republic of Korea. ${ }^{4}$ Department of Mechanical Engineering, Korea Polytechnic University, 237 Sangidaehak-Ro, Siheung-si, Gyeonggi-do, 15073, Republic of Korea. *email: omsnam@yuhs.ac
} 


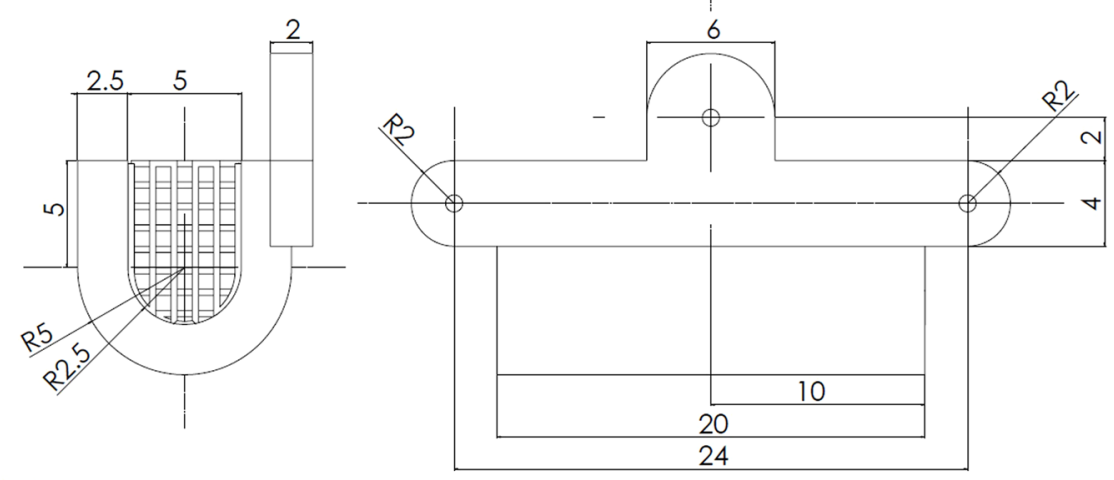

(a)

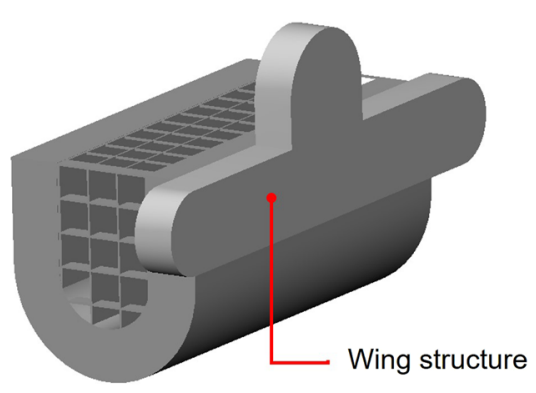

(b)

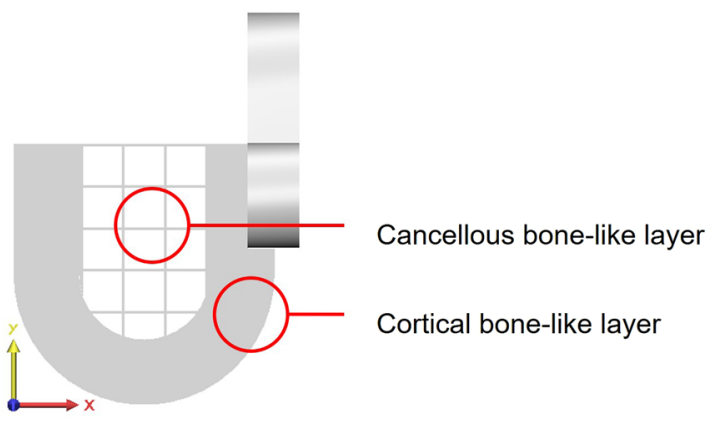

(c)

Figure 1. Design of 3D-printed PCL/ $\beta$-TCP scaffolds. (a) dimension, (b) wing structure for screw fixation, (c) different porosities.

to the best of our knowledge, there are few studies using 3D-printed PCL/ $\beta$-TCP scaffolds in mandibular reconstruction with a large defect model.

In this study, we suggest a novel design of PCL/ $\beta$-TCP scaffolds for mandibular reconstruction. The 3D-printed PCL/ $\beta$-TCP scaffolds were successfully fabricated by 3DP technology using a multi-head deposition system (MHDS). We assess the feasibility of 3D-printed PCL/ $\beta$-TCP scaffolds for a critical-sized defect (CSD) of canine mandible.

\section{Material and Methods}

Experimental animals and materials. This study was designed as a controlled preclinical study involving eight male, adult beagle dogs. The dogs were aged $12-15$ months and with a mean body weight of $12.5 \mathrm{~kg}$. They had no systemic disease and showed intact dentition and healthy maxillofacial appearance. Each animal was maintained in an individual cage under standard laboratory environment and were provided with a prescription diet (a/d Urgent Care, Hill's, Topeka, KS, USA) by a veterinarian and a standard laboratory pellet diet (Purina Canine LabDiet, Cargill, Dangjin, Republic of Korea). The animal selection, acclimatization, surgical protocols and management were approved by the Institutional Animal Care and Use Committee of Yonsei University Health System, Seoul, Korea (IACUC No. 2017-0326) and performed in accordance with the relevant guidelines and regulations.

Design of PCL/ $\beta$-TCP scaffold. For immobilization of scaffolds in mandibular defect, annexed wing structures containing three screw holes were designed for fixation. Wings are built up on three interfaces with residual bone (mesial, distal and alveolus) (Fig. 1a,b). The scaffold structure consists of two layers of different density (Fig. 1c). The external layer, comparable to the cortical bone, is composed of a dense layer with high porosity (70-75\%) and pore size greater than $300 \mu \mathrm{m}$, appropriate for maintaining the strength of scaffold and in vivo vascularization. The internal layer, comparable to the cancellous bone, is composed of a loose grid of which diameter is greater than $600 \mu \mathrm{m}$ for rapid bone regeneration from residual bone (Fig. 2a,b,c).

Fabrication PCL/ß-TCP scaffold using 3DP technology. PCL (PC 12, Corbion Purac, Gorinchem, Netherlands) and $\beta$-TCP (average diameter, $100 \mathrm{~nm}$; 7758-874, Premier Biomaterials, Tipperary, Ireland) were blended with a melting process. PCL was placed in a glass container and melted by heating for $15 \mathrm{~min}$ at $110^{\circ} \mathrm{C}$. After powdered $\beta$-TCP was added, PCL and the powdered $\beta$-TCP mixture were blended by hand for $10 \mathrm{~min}$. The $\mathrm{PCL} / \beta$-TCP mixture was transferred to a $10 \mathrm{ml}$ steel syringe in the MHDS and maintained at $120^{\circ} \mathrm{C}$. MHDS was operated using computer-aided manufacturing software. The PCL/ $\beta$-TCP material was extruded and stacked 4 times. The line width, pore size, and line height within the scaffolds were 300,400 , and $100 \mu \mathrm{m}$, respectively. The scaffolds had a rectangular pore architecture and a porosity of $57 \%$ as determined by $3 \mathrm{D}$ modeling software 

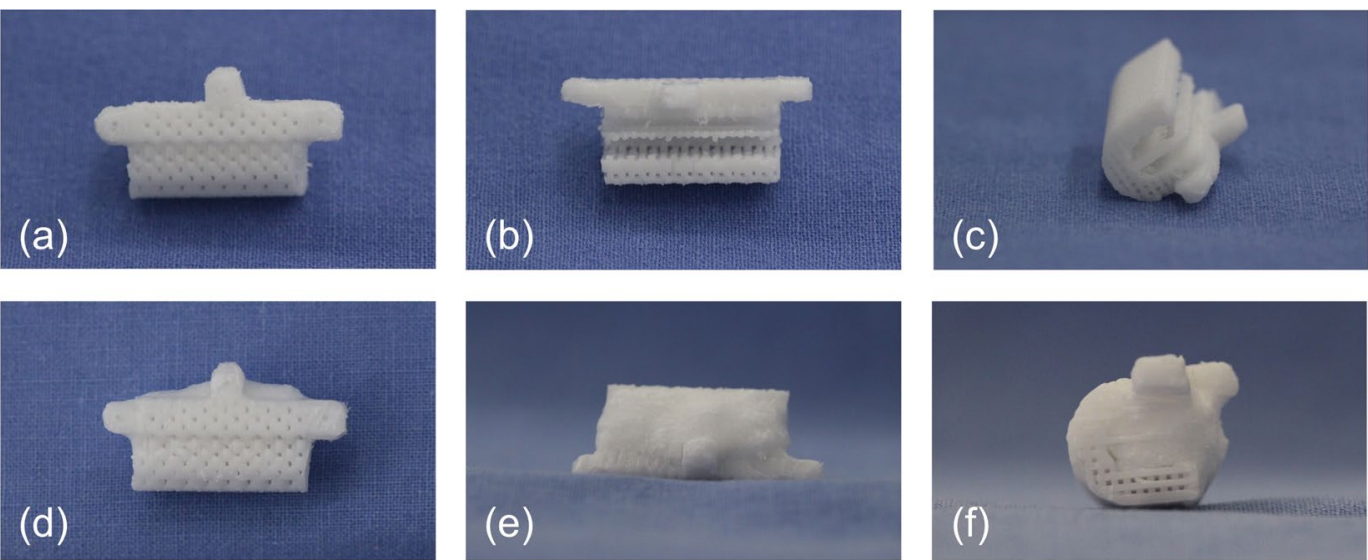

Figure 2. Morphology of 3D-printed PCL/ $\beta$-TCP scaffolds. PCL/ $\beta$-TCP scaffold (a), sagittal view; (b), axial view; (c) coronal view) and PCL/ $\beta$-TCP scaffold loaded with rhBMP-2 (d), sagittal view; (e), axial view; (f) coronal view).

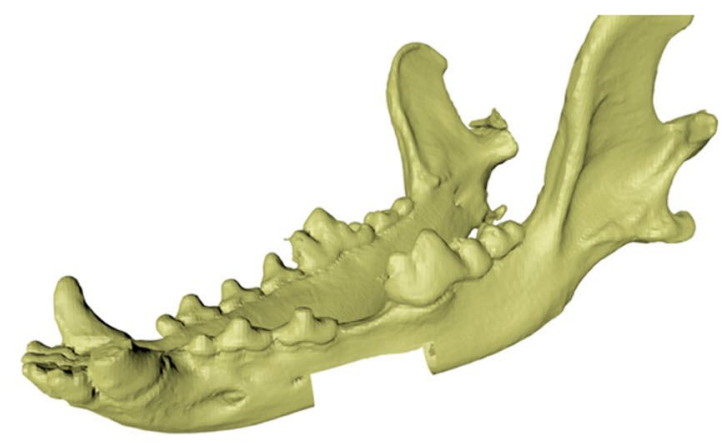

(a)

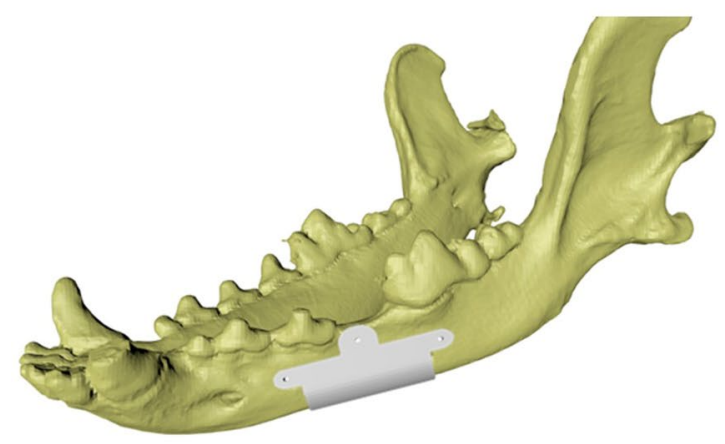

(b)

Figure 3. Modeling process of 3D-printed PCL/ $\beta$-TCP scaffolds. (a) $20 \times 10 \times 10 \mathrm{~mm}$ critical-sized defect was prepared, (b) 3D-printed scaffold was designed.

(3-Matic Research 9.0, Materialise, Leuven, Belgium) (Fig. 3). Pores were completely interconnected. The scaffolds were freeze-dried at $-85^{\circ} \mathrm{C}$ for 24 hours, then sterilized under a $450 \mathrm{~W}$ UV lamp for 4 hours.

The recombinant human bone morphogenetic protein-2 (rhBMP-2) loaded scaffolds were prepared for the PCL/TCP/BMP2 group as follows: $50 \mu \mathrm{g} / \mathrm{ml} \mathrm{rhBMP}-2$ solution (Cowellmedi, Busan, Republic of Korea) was 


\begin{tabular}{|l|l|}
\hline Group & Composition of scaffold \\
\hline Control & No scaffold \\
\hline PCL/TCP & PCL $(70 \mathrm{wt} \%) / \beta$-TCP $(30 \mathrm{wt} \%)$ \\
\hline PCL/TCP/BMP2 & PCL $(70 \mathrm{wt} \%) / \beta-\mathrm{TCP}(30 \mathrm{wt} \%) / \mathrm{rhBMP}-250 \mu \mathrm{g} / \mathrm{ml}$ \\
\hline PCL/TCP/ABP & $\mathrm{PCL}(70 \mathrm{wt} \%) / \beta-\mathrm{TCP}(30 \mathrm{wt} \%) /$ autogenous bone particles \\
\hline
\end{tabular}

Table 1. Characteristics of experimental groups $(n=4$, respectively).
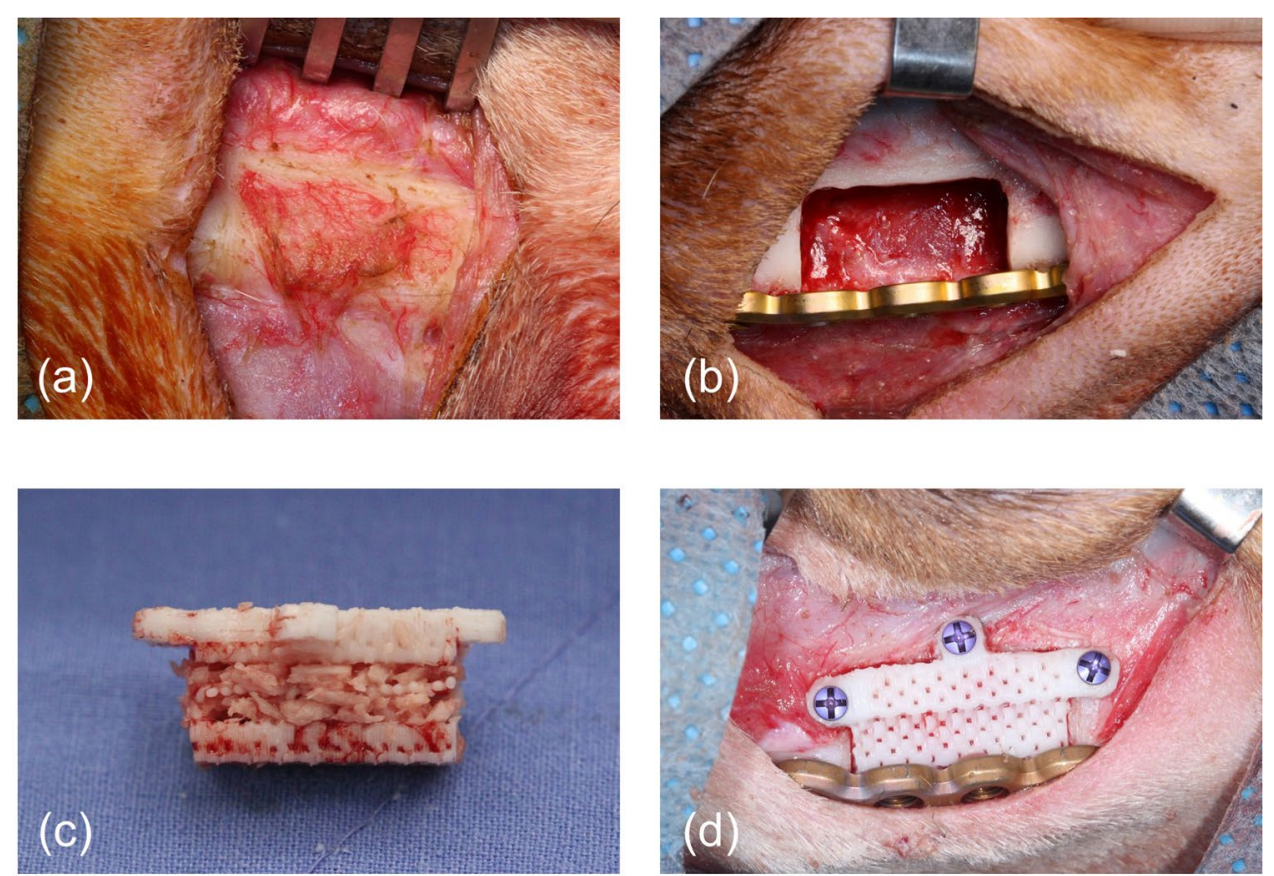

Figure 4. Surgical procedures. (a) periosteum left on bone surface to be simultaneously resected, (b) a criticalsized defect of mandible in control group, (c) PCL/ $\beta$-TCP scaffold with particularized autogenous bone in PCL/ TCP/ABP group, (d) implantation of scaffold and reinforcement of mandible with reconstruction plate in PCL/ TCP/BMP2 group.

mixed in 3\% collagen solution (MS Biotech, Republic of Korea). 3D-printed PCL/ $\beta$-TCP scaffold was immersed in the mixed solution. For penetration of mixed solution to the internal pores of scaffold, the mixed solution containing scaffold was centrifuged at $1000 \mathrm{rpm}$. The rhBMP-2/collagen-infiltrated scaffold was then freeze-dried at $-85^{\circ} \mathrm{C}$ and maintained at $-4^{\circ} \mathrm{C}$ (Fig. 2 d,e,f).

Study design. Four experimental groups were constituted at bilateral mandible in eight beagle dogs in this study ( $\mathrm{n}=4$, respectively) as follows: a control group (control, $\mathrm{n}=4), \mathrm{PCL} / \beta$-TCP scaffold only group (PCL/TCP, $\mathrm{n}=4), \mathrm{PCL} / \beta$-TCP scaffold + rhBMP-2 $50 \mu \mathrm{g} / \mathrm{ml}(\mathrm{PCL} / \mathrm{TCP} / \mathrm{BMP} 2, \mathrm{n}=4)$ and PCL/ $\beta$-TCP scaffold with autogenous bone particles ( $\mathrm{PCL} / \mathrm{TCP} / \mathrm{ABP}, \mathrm{n}=4)$ (Table 1$)$. No scaffolds were placed into the defect for the control group. In the PCL/TCP/BMP2 group, at a concentration of $50 \mu \mathrm{g} / \mathrm{ml}, \mathrm{rhBMP}-2$ with a collagen sponge as a carrier was loaded into the scaffolds For the PCL/TCP/ABP group, autogenous bone obtained from mandibular resection was particularized with a bone crusher and inserted into the loose internal structure of scaffolds (Fig. 4c). The sample size was determined by Mead's resource equation.

Surgical procedures. Oral prophylaxis was applied to all experimental animals prior to the surgical procedures. The tooth extraction was performed on first molars and premolars in bilateral mandible. After alfaxalone induction $(0.2 \mathrm{mg} / \mathrm{kg}$ intravenous injection; Hans Nieuwendijk. Nederland), animals were anaesthetized with isoflurane (Choongwae Co., Seoul, Republic of Korea). Then, $1.8 \mathrm{~mL}$ of 2\% lidocaine HCL with 1:100,000 epinephrine (Huons, Seongnam-si, Gyeonggi-do, Republic of Korea) was injected for infiltrative anesthesia on intraoral mucosa of premolar and molar area. The 4th premolar and 1st molar were sectioned into several parts into mesial and distal roots. After buccal and lingual walls of the extraction socket were preserved intact, the socket was closed with synthetic absorbable suture (3-0 coated VICRYL Plus, Ethicon Inc., NJ, USA) for proper healing. Animals were allowed a two-month recovery period.

2 months from the extraction of the 4 th premolar and 1st molar, bone defects were created on bilateral mandible of all 8 beagles. Under general anesthesia as detailed above, local anesthesia was applied on the submandibular skin area. The mandible was exposed via an extraoral submandibular approach. At the level of inferior border 
in the mandibular body, a rectangular-shaped critical-sized defect (CSD) was outlined with a surgical marking pencil to be $20 \mathrm{~mm}$ in length, $10 \mathrm{~mm}$ in width, and $10 \mathrm{~mm}$ in height $(20 \times 10 \times 10 \mathrm{~mm})$. The en bloc resection of inferior border and periosteum was performed bicortically with reciprocating saw (Fig. 4a). The inferior alveolar neurovascular bundle was ligated. The scaffolds were then implanted into 12 defects in 6 beagles ( 4 defects in 2 beagles were left empty as negative control) and fixed with $1.95 \mathrm{~mm}$-diameter self-drilling \& tapping screws (Optimus Maxillofacial Plating System, Osteonic, Seoul, Republic of Korea) on three interfaces with residual bone (mesial, distal and alveolus). Following the fixation of scaffolds, osteosynthesis was then performed using a $2.4 \mathrm{~mm}$ thick titanium reconstruction plate (Optimus Mandible Plating System, Osteonic, Seoul, Republic of Korea) for $2.7 \mathrm{~mm}$-diameter cortical screws (Fig. 4b,d). After copious saline irrigation, the subcutaneous layer was closed with synthetic absorbable suture (3-0 coated VICRYL Plus, Ethicon Inc., NJ, USA). Skin was closed using synthetic non-absorbable monofilament suture (3-0 Dafilon, B.Braun Surgical S.A., Barcelona, Spain). At 12 weeks after implantation, animals were euthanized with intravenous injection of concentrated potassium chloride (Daejung, Sigeung, Korea). After absence of cardiovascular function was verified, block sections including implants, alveolar bone, and surrounding mucosa were collected.

Micro-computed tomographic analysis. Microcomputed tomographic images were made using a microcomputed tomography scanner (Skyscan 1076; Skyscan, Kontich, Belgium) at a tube voltage of $100 \mathrm{kV}$ and a tube current of $100 \mu \mathrm{A}$ with rotation steps of $0.5^{\circ}$ over a trajectory of $360^{\circ}$. The region of interest (ROI) was defined as a regular cuboid with $1 \mathrm{~cm}$ length, $5 \mathrm{~mm}$ width and $1 \mathrm{~cm}$ height including the internal scaffold space. Image-analysis programs (CTAn 1.12.0.0 and CTVol 2.2.1.0; Skyscan) were used for morphometric analysis and rendering. Mineralized bone volume was calculated by grayscale index from 39 to 52 (defined as radiopaque tissue) in inverse images. Bone volume/tissue volume (BV/TV, mm3), trabecular numbers (Tb.N, 1/mm), trabecular thickness (Tb.Th, $\mathrm{mm}$ ), and trabecular separation (Tb.Sp, $\mathrm{mm}$ ) of each group were assessed within ROI. (Supplementary Fig. S1)

Histological and histomorphometric analysis. Tissues containing the scaffolds were removed en bloc and fixed in $4 \%$ neutral-buffered formaldehyde, then dehydrated using an ascending series of alcohol concentrations (80-100\%) and embedded in methyl methacrylate (Technovit 7200; Exakt Apparatebau, Norderstedt, Germany) for undecalcified sectioning. The sections containing the whole scaffolds along the sagittal plane were produced at $200 \mu \mathrm{m}$ using a macro-cutting, then made at a final thickness of $20 \mu \mathrm{m}$ using a grinding system (Exakt 310 CP series; Exakt Apparatebau, Norderstedt, Germany). The sections were stained with hematoxylin-eosin (H\&E) and Goldner's Masson trichrome separately.

The histologic sections were examined using a light microscope (BX51; Olympus, Tokyo, Japan) to identify newly formed bone (NB), residual scaffold (RS) and nonmineralized tissue (NMT) area under 50 $\times$ magnification. Area measurements were made using KAPPA ImageBase (Kappa Optronics GmbH, Gleichen, Germany) and Image J (Image J, NIH, USA). The rectangular area of interest (ROI) was defined on the anterior and posterior interfaces between scaffold and residual bone. The ratios of the NB, RS and NMT to the total area were calculated as a percentage.

Statistical analysis. Quantitative data were expressed as the mean \pm standard deviation. Wilcoxon rank-sum test and Kruskal-Wallis test with the Tukey significant difference post hoc test were also used to assess difference among the groups. All analyses were performed using IBM SPSS Statistics for Windows, version 22.0 (IBM Corp., Armonk, NY, USA). A probability value (two-tailed) of less than 0.05 was considered to indicate statistical significance $(\mathrm{P}<0.05)$.

\section{Results}

Clinical observations. All the animals presented with favorable general status. Except or one PCL/TCP group sample, the remaining 15 samples demonstrated no detectable wound dehiscence or scaffold exposure throughout the experiment. The one scaffold in the PCL/TCP group showed intraoral exposure of the wing structure on the mesial area of defect on postoperative day 7. After intensive care on the intraoral wound for the remaining follow-up period, no abnormalities were noticed. All the obtained samples were included in the analyses.

Micro-computed tomographic analysis. Volumetric measurement results were obtained using micro-CT (Fig. 5, Supplementary Fig. S2 and Table S1). At 12 weeks after surgery, new bone volumes (mean \pm standard deviation, $\mathrm{mm} 3$ ) in the groups were $33.46 \pm 21.81,30.50 \pm 16.26,97.17 \pm 28.11$ and $68.32 \pm 21.47$, respectively. The differences between some groups were significant $(\mathrm{P}<0.05)$. Group 3, which was loaded with rhBMP-2, showed the highest new bone volume ( $\mathrm{mm} 3)$.

Histologic observations and histomorphometric analysis. Histological findings are shown in Fig. 6. Scaffolds were properly positioned in mandibular defects. At 12 weeks after surgery, calcification and de novo formation were observed in the experimental groups. In the PCL/TCP group, small amounts of new bone grew into the PCL/ $\beta$-TCP scaffold and interface region between scaffold and residual bone. More newly formed bone was observed in PCL/TCP/BMP2 and PCL/TCP/ABP groups than in control and PCL/TCP groups. In particular, the loose porous area in the PCL/TCP/BMP2 and PCL/TCP/ABP groups had been filled by new bone and fibrous connective tissues. Abnormal resorption or degradation of the scaffold structure was not observed in any group.

Histomorphometric results are summarized in Table 2. At 12 weeks after surgery, newly formed bone (\%) in the control, PCL/TCP, PCL/TCP/BMP2 and PCL/TCP/ABP groups were $22.70 \pm 4.24,25.78 \pm 20.94,33.27 \pm 9.64$ and $33.32 \pm 14.66$ respectively. Newly formed bone at interfaces was highest in the PCL/TCP/BMP2 group and 
(a)

\section{Control}
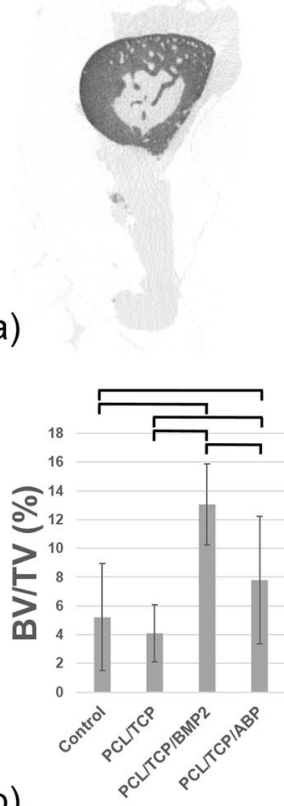

(b)

(c)

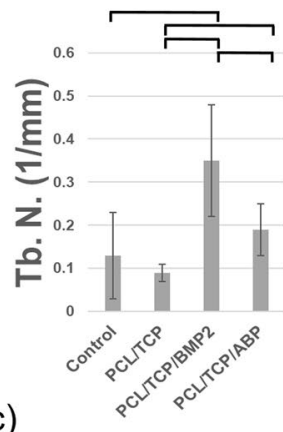

PCL/TCP/BMP2
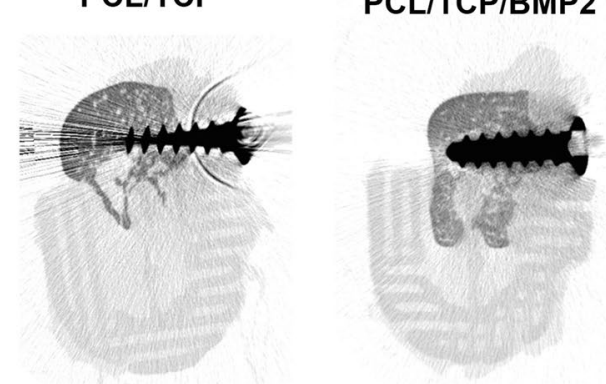

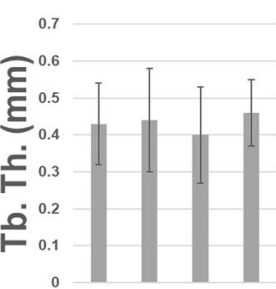

(d)

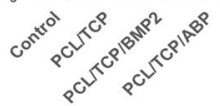

\section{PCL/TCPIABP}

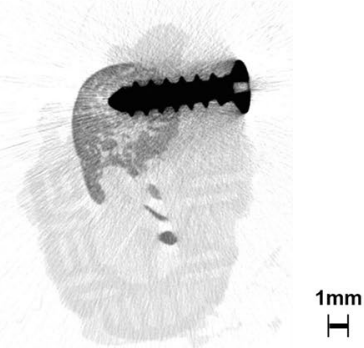

(e)

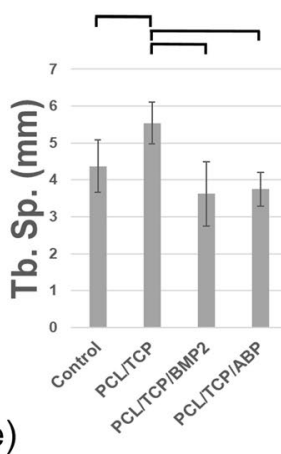

Figure 5. De novo bone formation in micro-computed tomography. (a) Coronal view of micro-computed tomography, (b) bone volume-total volume ratio (BV/TV), (c) trabecular number (Tb. N.), (d) trabecular thickness (Tb. Th.), (e) trabecular separation (Tb. Sp.). Solid line indicates statistically significant difference between groups.
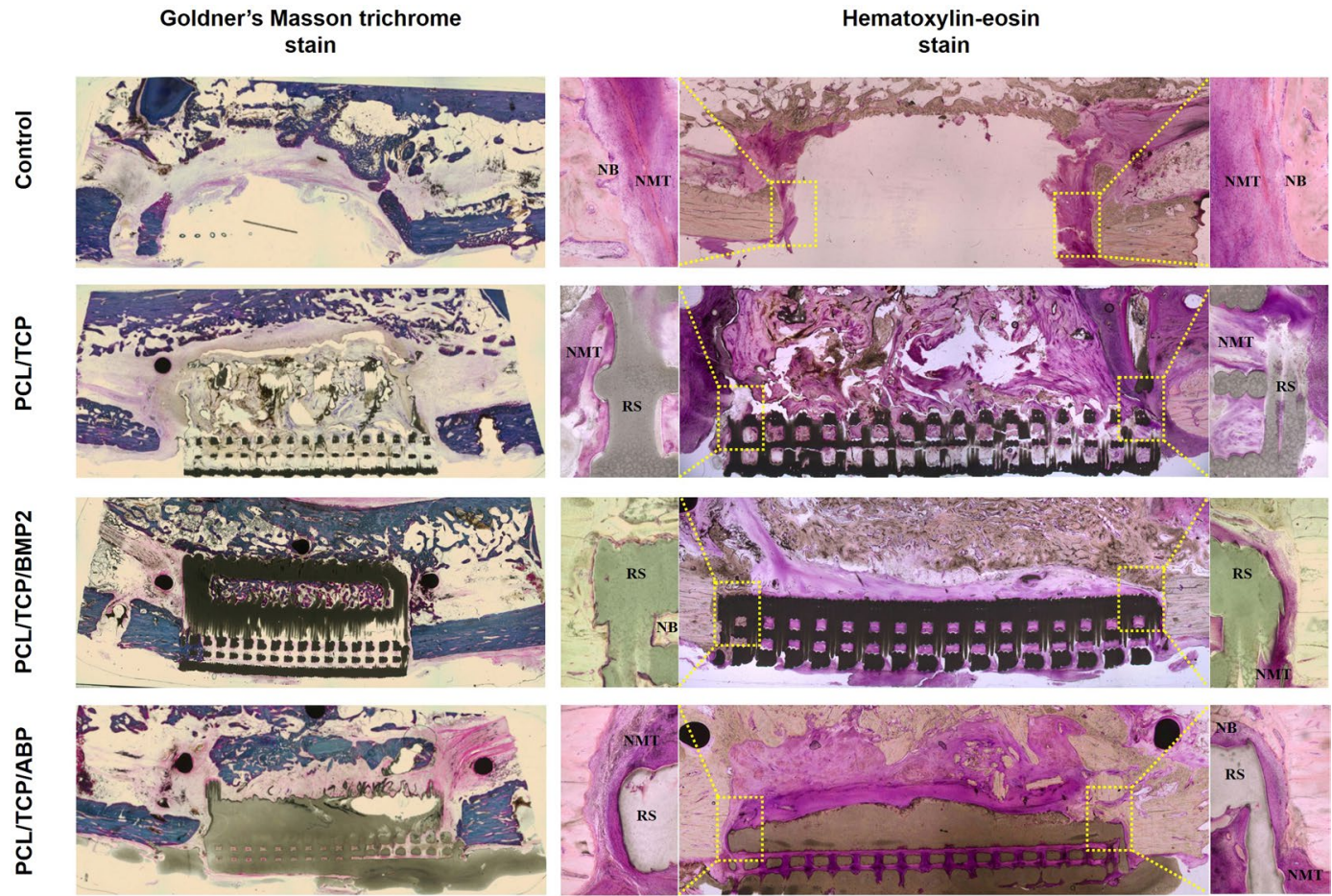

Figure 6. Histologic sections (original magnification $10 \times$ ). In the left column, the Goldner's Masson trichrome stained results were shown. The hematoxylin-eosin stain results were presented in right column. ROI was shown in yellow dotted areas and corresponding regions were magnified $(100 \times)$. 


\begin{tabular}{|l|l|l|l|l|l|}
\hline Parameter & Control & PCL/TCP & PCL/TCP/BMP2 & PCL/TCP/ABP & $P$ value \\
\hline NB & $22.70 \pm 4.24$ & $25.78 \pm 20.94$ & $33.27 \pm 9.64$ & $33.32 \pm 14.66$ & 0.052 \\
\cline { 1 - 3 }$(\%)$ & NA & $31.69 \pm 7.58$ & $33.31 \pm 10.13$ & $32.07 \pm 12.14$ & 0.717 \\
\hline RS & $77.30 \pm 4.24$ & $42.53 \pm 14.71$ & $33.41 \pm 9.50$ & $34.60 \pm 5.64$ & $0.012 *$ \\
\hline NMT & & & & &
\end{tabular}

Table 2. Histomorphometric analysis within area of interest $(n=4$, respectively, mean $\pm S D)$. Abbreviation: NB, newly formed bone; RS, residual scaffold; NA, not applicable; NMT, nonmineralized tissue (*p $<0.05)$.

lowest in the control group. A trend towards more bone formation was observed in PCL/TCP/BMP2 and PCL/ TCP/ABP groups, but the results lacked statistical significance $(\mathrm{P}=0.052)$.

\section{Discussion}

Our in vivo study demonstrated the benefits of 3D-printed PCL/ $\beta$-TCP scaffolds in mandibular reconstruction. First, the design of scaffold could increase the rigidity of fixation to the residual bone. Previous in vivo studies used different fixation modalities to immobilize the scaffold in large defect. Researchers used dental implants ${ }^{13}$, mini screws through the PCL/TCP scaffold ${ }^{14}$ or an endo-prosthetic design of the PCL/TCP scaffold into the mandibular defect of monkey ${ }^{15}$. In this study, we designed less invasive modality with three semicircular shaped, $4.0 \mathrm{~mm}$ diameter $\times 2.0 \mathrm{~mm}$ thickness external wing structures containing guide holes for mini-screw fixation. This was to inhibit unpredictable damage to the scaffold, diminution of $\mathrm{x}$-ray scattering during micro-computed tomographic analysis, and to ensure less damage to the adjacent structure and distinct quantification of the de novo bone formation within scaffold. For 12 weeks after implantation of the scaffold, the screw fixations were favorably maintained in the residual bone. Those external wing structures could also be customized for individual geometry of the defect and extended for rigid fixation of mandibular discontinuity defect. Second, MHDS enabled the fabrication of PCL/ $\beta$-TCP scaffolds with double porous layer. Through the bimodal macro-porosities, we could provide the PCL/ $\beta$-TCP scaffolds with mechanical support from the cortical bone-like layer of which diameter is approximately $300 \mu \mathrm{m}$ and enhance the new bone growth from residual bone into the cancellous bone-like layer of which diameter is over $600 \mu \mathrm{m}$. The present study revealed acceptable outcomes of new bone growth from the alveolus into the cancellous bone-like layer in the canine mandible. Those new bone that connect both ends of the defect could compensate loss of mechanical strength due to degradation of PCL/ $\beta$-TCP scaffolds.

The mechanical property of PCL/ $\beta$-TCP scaffold might be insufficient to be applied alone in the reconstruction of human mandible. According to Bao et al., the hydroxyapatite/polycaprolactone (HA/PCL) scaffolds were fabricated with biomimetic structure of which proper osseointegration and mechanical property was observed in rabbit tibia ${ }^{16}$. They used titanium miniplate fixation to stabilize the HA/PCL scaffolds in the weight bearing bone. Similar to the mechanical property of the HA/PCL scaffolds, the compressive strength of PCL/ $\beta$-TCP scaffolds has been verified as approximately $20 \mathrm{MPa}$, which is 2 times higher than that of cancellous bone and one tenth of cortical bone in mandible ${ }^{17,18}$ (Material, methods and results are detailed in Supplementary Discussion S1 and Supplementary Fig. S3). In the clinical situation, reconstructive surgeons should consider the biomechanics of mandible including tension, compression and torsion ${ }^{18}$. For distribution of loading forces to the mandible, the titanium plate with load-bearing fixation method should be combined for mandibular reconstruction using 3D-printed PCL/ $\beta$-TCP scaffolds.

The classic definition of CSD is the smallest size defect that will not heal over the lifetime of the animal or a defect which shows less than 10 percent bone regeneration during the first year of defect healing ${ }^{19-22}$. Defects larger than CSD result in non-healing with bone regeneration and fibrous tissue engaging in the defects. To date, only a few studies have documented the CSD in the canine mandible $e^{23,24}$. According to Huh et al., after removal of the periosteum in mongrel dogs, bony bridges were not observed in mandibular defects more than $15 \mathrm{~mm}$ size at 6 months after segmental mandibulectomy. They suggested the critical-sized defect in canine mandible is $50 \mathrm{~mm}$ in presence of periosteum and $15 \mathrm{~mm}$ in absence of periosteum. We performed the mandibular resection including periosteum with size of $20 \times 10 \times 10 \mathrm{~mm}$ to create a CSD in beagle dogs. Previous studies for PCL/ TCP scaffold used similar dimensions as a CSD in canine mandible. Rai et al. created a CSD of $18 \times 10 \times 7 \mathrm{~mm}$ in frontal and caudal mandible of mongrel dogs ${ }^{13}$. Using CSD of $20 \times 10 \times 10 \mathrm{~mm}$ in buccal cortical plate of canine mandible, A. Khojasteh et al. reported the bone regeneration of PCL/TCP scaffold with BMSC ${ }^{14}$.

In this study design, there was a need to limit the use of rhBMP-2. Since the potential carcinogenicity of rhBMP-2 in association with oral squamous cell carcinoma (OSCC) remains controversial ${ }^{25-29}$. Gao et al. suggest that BMP-2 may not have adverse effects on OSCC, as measured by proliferation and angiogenesis ${ }^{25}$. However, human OSCC cell lines have been shown to express BMP-2 and be associated with rapid tumor growth in orthotopic animal model ${ }^{27}$. Although there is no agreement whether rhBMP-2 promotes, inhibits, or has no association with tumorigenesis of OSCC, maxillofacial surgeons should be cautious in applying rhBMP-2 in malignant tumor patients. In clinical situations, a PCL/ $\beta$-TCP scaffold combined with autogenous bone particle is applicable for mandibular defect after malignant tumor resection. Meanwhile, a rhBMP-2 loaded PCL/ $\beta$-TCP scaffold can be applied in mandibular defect derived from trauma, osteomyelitis, medication-related osteonecrosis of the jaw and particular benign lesions (ameloblastoma, odontogenic keratocyst, giant cell granuloma and etc.) ${ }^{2}$.

Our study has several limitations. The volume of new bone formation was insufficient for clinical application in mandibular reconstruction. In the present study, we also used the periosteal resection model to evaluate the 
efficacy of PCL/ $\beta$-TCP scaffold with low dose of rhBMP-2. Although the PCL/TCP/BMP2 group showed no significant difference in the volume of new bone formation compared with PCL/TCP/ABP groups, the absolute volume of new bone formation was remarkably lower than that of previous studies. To exclude the healing potential of periosteum and assess the bone regeneration efficacy of scaffolds, previous studies reported in vivo results in a periosteal resection model. In the absence of periosteum, researchers documented that bone regeneration with scaffold alone might be challenging ${ }^{30-32}$. To achieve clinically acceptable results, further studies are needed to determine the adequate concentration of rhBMP- 2 for periosteal resection model in mandibular discontinuity defect. Another limitation is that degradation period of PCL/ $\beta$-TCP scaffold could not be assessed at 12 weeks after surgery. Previous studies demonstrated initiation of in vitro degradation within 28 days in simulated body fluids ${ }^{33}$, remarkable in vivo degradation at 24 weeks after implantation in rat abdomen ${ }^{34}$, and about $33 \%$ in vivo degradation of PCL/ $\beta$-TCP scaffold from 6 to 9 months after implantation in canine mandible ${ }^{13}$. More extensive investigation is necessary for long term observation on degradation of scaffold and associated inflammatory response.

This study was conducted to evaluate the feasibility of 3D-printed PCL/ $\beta$-TCP scaffolds in large defect of canine mandible. The scaffold incorporated heterogeneous pore sizes for rapid bone ingrowth and additional wing structures for more stable screw fixation. Within the limitations of the present study, 3D-printed PCL/ $\beta$-TCP scaffolds showed acceptable potential for mandibular reconstruction. For future clinical implementation, a larger study of PCL/ $\beta$-TCP scaffolds with high dose of growth factors is required.

Received: 9 September 2019; Accepted: 3 March 2020;

Published online: 18 March 2020

\section{References}

1. Choi, S. W., Kim, H. J., Koh, K. S., Chung, I. H. \& Cha, I. H. Topographical anatomy of the fibula and peroneal artery in Koreans. Int. J. Oral. Maxillofac. Surg. 30, 329-332 (2001).

2. Desai, S. C., Sclaroff, A. \& Nussenbaum, B. Use of recombinant human bone morphogenetic protein 2 for mandible reconstruction. JAMA Facial Plast. Surg. 15, 204-209 (2013).

3. Rasperini, G. et al. 3D-printed Bioresorbable Scaffold for Periodontal Repair. J. Dent. Res. 94, 153S-157S (2015).

4. Ho Han, H. et al. Orbital wall reconstruction in rabbits using 3D printed polycaprolactone- $\beta$-tricalcium phosphate thin membrane. Mater. Lett. 218, 280-284 (2018).

5. Park, S. A. et al. In Vivo Evaluation of 3D-Printed Polycaprolactone Scaffold Implantation Combined with beta-TCP Powder for Alveolar Bone Augmentation in a Beagle Defect Model. Materials (Basel) 11 (2018).

6. Dupret-Bories, A., Vergez, S., Meresse, T., Brouillet, F. \& Bertrand, G. Contribution of 3D printing to mandibular reconstruction after cancer. Eur. Ann. otorhinolaryngology, head. neck Dis. 135, 133-136 (2018).

7. Shim, J. H. et al. Fabrication of blended polycaprolactone/poly(lactic-co-glycolic acid)/beta-tricalcium phosphate thin membrane using solid freeform fabrication technology for guided bone regeneration. Tissue Eng. Part. A 19, 317-328 (2013).

8. Bae, E. B. et al. Efficacy of rhBMP-2 Loaded PCL/beta-TCP/bdECM Scaffold Fabricated by 3D Printing Technology on Bone Regeneration. Biomed. Res. Int. 2018, 2876135 (2018).

9. Shim, J. H. et al. Efficacy of rhBMP-2 loaded PCL/PLGA/beta-TCP guided bone regeneration membrane fabricated by 3D printing technology for reconstruction of calvaria defects in rabbit. Biomed. Mater. 9, 065006 (2014).

10. Won, J. Y. et al. Evaluation of 3D printed PCL/PLGA/beta-TCP versus collagen membranes for guided bone regeneration in a beagle implant model. Biomed. Mater. 11, 055013 (2016).

11. Han, H. H. et al. Reconstruction of Complex Maxillary Defects Using Patient-specific 3D-printed Biodegradable Scaffolds. Plastic and reconstructive surgery. Glob. open. 6, e1975-e1975 (2018).

12. Kim, S. E., Shim, K. M., Jang, K., Shim, J. H. \& Kang, S. S. Three-Dimensional Printing-based Reconstruction of a Maxillary Bone Defect in a Dog Following Tumor Removal. Vivo 32, 63-70 (2018).

13. Rai, B. et al. Polycaprolactone-20\% tricalcium phosphate scaffolds in combination with platelet-rich plasma for the treatment of critical-sized defects of the mandible: a pilot study. J. Oral. Maxillofac. Surg. 65, 2195-2205 (2007).

14. Khojasteh, A. et al. The effect of PCL-TCP scaffold loaded with mesenchymal stem cells on vertical bone augmentation in dog mandible: a preliminary report. J. Biomed. Mater. Res. B Appl. Biomater. 101, 848-854 (2013).

15. Chanchareonsook, N. et al. Segmental mandibular bone reconstruction with a carbonate-substituted hydroxyapatite-coated modular endoprosthetic poly(varepsilon-caprolactone) scaffold in Macaca fascicularis. J. Biomed. Mater. Res. B Appl. Biomater. 102, 962-976 (2014).

16. Bao, X. et al. 3D biomimetic artificial bone scaffolds with dual-cytokines spatiotemporal delivery for large weight-bearing bone defect repair. Sci. Rep. 7, 7814 (2017).

17. Misch, C. E., Qu, Z. \& Bidez, M. W. Mechanical properties of trabecular bone in the human mandible: implications for dental implant treatment planning and surgical placement. J Oral Maxillofac Surg 57, 700-706; discussion 706-708 (1999).

18. van Eijden, T. M. Biomechanics of the mandible. Crit. Rev. oral. Biol. medicine: an. Off. Publ. Am. Assoc. Oral. Biologists 11, 123-136 (2000).

19. Gugala, Z. \& Gogolewski, S. Regeneration of segmental diaphyseal defects in sheep tibiae using resorbable polymeric membranes: a preliminary study. J. orthopaedic trauma. 13, 187-195 (1999).

20. Reichert, J. C. et al. The challenge of establishing preclinical models for segmental bone defect research. Biomater. 30, 2149-2163 (2009).

21. Shah, S. R. et al. A composite critical-size rabbit mandibular defect for evaluation of craniofacial tissue regeneration. Nat. Protoc. 11, 1989-2009 (2016).

22. Marei, H. F., Mahmood, K. \& Almas, K. Critical Size Defects for Bone Regeneration Experiments in the Dog Mandible: A Systematic Review. Implant. Dent. 27, 135-141 (2018).

23. Schmitz, J. P. \& Hollinger, J. O. The critical size defect as an experimental model for craniomandibulofacial nonunions. Clinical orthopaedics and related research, 299-308 (1986).

24. Huh, J. Y. et al. Critical size defect in the canine mandible. Oral. Surg. Oral Med. Oral Pathol. Oral Radiol. Endod. 100, 296-301 (2005).

25. Gao, Q. et al. Effects of bone morphogenetic protein-2 on proliferation and angiogenesis in oral squamous cell carcinoma. Int. J. Oral. Maxillofac. Surg. 39, 266-271 (2010).

26. Devine, J. G., Dettori, J. R., France, J. C., Brodt, E. \& McGuire, R. A. The use of rhBMP in spine surgery: is there a cancer risk? Evidence-based spine-care J. 3, 35-41 (2012).

27. Kokorina, N. A., Lewis, J. S. Jr., Zakharkin, S. O., Krebsbach, P. H. \& Nussenbaum, B. rhBMP-2 has adverse effects on human oral carcinoma cell lines in vivo. Laryngoscope 122, 95-102 (2012). 
28. Kim, M. J., Kim, K. M., Kim, J. \& Kim, K. N. BMP-2 promotes oral squamous carcinoma cell invasion by inducing CCL5 release. PLoS One 9 , e108170 (2014).

29. Skovrli, B. et al. Association Between BMP-2 and Carcinogenicity. Spine 40, 1862-1871 (2015).

30. Yuan, J., Cui, L., Zhang, W. J., Liu, W. \& Cao, Y. Repair of canine mandibular bone defects with bone marrow stromal cells and porous beta-tricalcium phosphate. Biomater. 28, 1005-1013 (2007).

31. Liu, X. et al. Mesenchymal stem cells systemically injected into femoral marrow of dogs home to mandibular defects to enhance new bone formation. Tissue Eng. Part. A 20, 883-892 (2014).

32. Liu, X. et al. Osteoprotegerin gene-modified BMSCs with hydroxyapatite scaffold for treating critical-sized mandibular defects in ovariectomized osteoporotic rats. Acta Biomater. 42, 378-388 (2016).

33. Lei, Y., Rai, B., Ho, K. H. \& Teoh, S. H. In vitro degradation of novel bioactive polycaprolactone $-20 \%$ tricalcium phosphate composite scaffolds for bone engineering. Mater. Sci. Engineering: C. 27, 293-298 (2007).

34. Yeo, A., Rai, B., Sju, E., Cheong, J. J. \& Teoh, S. H. The degradation profile of novel, bioresorbable PCL-TCP scaffolds: an in vitro and in vivo study. J. Biomed. Mater. research. Part. A 84, 208-218 (2008).

\section{Acknowledgements}

This research was supported by the Basic Science Research Program through the National Research Foundation of Korea, funded by the Ministry of Education (NRF-2016R1D1A1A02936925).

\section{Author contributions}

S.L. and W.N. designed and performed the experiments. D.C. and J.-H.S. provided materials and fabricated the scaffolds. S.L. analyzed the data and wrote the main manuscript text. W.N. directed the whole process.

\section{Competing interests}

The authors declare no competing interests.

\section{Additional information}

Supplementary information is available for this paper at https://doi.org/10.1038/s41598-020-61944-w.

Correspondence and requests for materials should be addressed to W.N.

Reprints and permissions information is available at www.nature.com/reprints.

Publisher's note Springer Nature remains neutral with regard to jurisdictional claims in published maps and institutional affiliations.

(c) (i) Open Access This article is licensed under a Creative Commons Attribution 4.0 International License, which permits use, sharing, adaptation, distribution and reproduction in any medium or format, as long as you give appropriate credit to the original author(s) and the source, provide a link to the Creative Commons license, and indicate if changes were made. The images or other third party material in this article are included in the article's Creative Commons license, unless indicated otherwise in a credit line to the material. If material is not included in the article's Creative Commons license and your intended use is not permitted by statutory regulation or exceeds the permitted use, you will need to obtain permission directly from the copyright holder. To view a copy of this license, visit http://creativecommons.org/licenses/by/4.0/.

(C) The Author(s) 2020 